\title{
Mixed adenoendocrine carcinoma in the extrahepatic biliary tract: A case report and literature review
}

\author{
LIANG ZHANG ${ }^{1-3}$, ZHENGTAO YANG $^{1-3}$, QING CHEN $^{4}$, MENGXIA LI ${ }^{1-3}$, XIAOLU ZHU $^{1-3}$, \\ DALONG WAN ${ }^{1-3}$, HAIYANG XIE ${ }^{1-3}$ and SHUSEN ZHENG ${ }^{1-3}$
}

\begin{abstract}
${ }^{1}$ Division of Hepatobiliary and Pancreatic Surgery, Department of Surgery, First Affiliated Hospital, School of Medicine, Zhejiang University; ${ }^{2}$ Key Laboratory of Combined Multi-Organ Transplantation, National Health and Family Planning Commission (NHFPC); ${ }^{3}$ Key Laboratory of The Diagnosis and Treatment of Organ Transplantation, Chinese Academy of Medical Sciences (CAMS); ${ }^{4}$ Department of Pathology, First Affiliated Hospital, School of Medicine, Zhejiang University, Hangzhou, Zhejiang 310000, P.R. China
\end{abstract}

Received August 23, 2018; Accepted April 2, 2019

\section{DOI: $10.3892 / \mathrm{ol} .2019 .10502$}

\begin{abstract}
Neuroendocrine neoplasm (NEN) comprises a group of tumors that exhibit neuroendocrine phenotypes. NEN is subclassified into neuroendocrine tumor (NET), neuroendocrine carcinoma (NEC) and mixed adenoendocrine carcinoma (MANEC), based on histopathological parameters. NEN in the extrahepatic biliary tract (EHBT) is uncommon. Little is known about its clinicopathological features and prognostic indicators. The present study presented a case of MANEC in the distal common bile duct (CBD) and reviewed previous cases of NENs in the EHBT to characterize the clinical settings of this disease entity and to identify influencing factors of survival outcomes. A 64-year-old Chinese woman presented with abdominal pain and jaundice. Imaging studies demonstrated malignant stenosis in the distal CBD. Bile duct brush cytology revealed small clusters of atypical cells. Following an initial diagnosis of distal cholangiocarcinoma (CCA), the patient underwent pancreaticoduodenectomy. Histological analysis combined with immunohistochemical investigation of the resected specimen revealed a collision tumor that was
\end{abstract}

Correspondence to: Dr Haiyang Xie or Professor Shusen Zheng, Division of Hepatobiliary and Pancreatic Surgery, Department of Surgery, First Affiliated Hospital, School of Medicine, Zhejiang University, 79 Qingchun Road, Hangzhou, Zhejiang 310000, P.R. China E-mail: xiehy@zju.edu.cn

E-mail: shusenzheng@zju.edu.cn

Abbreviations: CBD, common bile duct; CCA, cholangiocarcinoma; EHBT, extrahepatic biliary tract; ERCP, endoscopic retrograde cholangiopancreatography; GCDFP-15, gross cystic disease fluid protein-15; IHC, immunohistochemical; IQR, interquartile range; MANEC, mixed adenoendocrine carcinoma; MMG, mammaglobin; NEC, neuroendocrine carcinoma; NEN, neuroendocrine neoplasm; NET, neuroendocrine tumor; TTF-1, thyroid transcription factor-1

Key words: neuroendocrine neoplasm, extrahepatic biliary tract, mixed adenoendocrine carcinoma, management, prognosis composed of poorly differentiated adenocarcinoma and NEC. Each histological component accounted for $>30 \%$ of the tumor. The definitive diagnosis was a MANEC in the distal CBD. Multiple intrahepatic and pulmonary metastases were observed postoperatively over 8 months. The patient succumbed to the disease 12 months after surgery. In conclusion, NEN occurs infrequently in the EHBT, with NET being the predominant type. NEN in the EHBT is extremely challenging to diagnose preoperatively due to its tendency to mimic CCA. Patients with NEN in the EHBT exhibited extremely distinct oncology outcomes according to pathological types. Additionally, old age ( $>60$ years) and the presence of tumor recurrence were associated with decreased survival of patients with NEN.

\section{Introduction}

Originating in various tissues, neuroendocrine neoplasm (NEN) comprises a family of tumor types that exhibit neuroendocrine differentiation characteristics, including neurosecretory granules, synaptic-like vesicles and production of different peptides $(1,2)$. Despite the shared neuroendocrine phenotypes, NENs are represented by an extremely heterogeneous entity of varied histopathological features (1). Therefore, NENs are divided into three main categories according to the 2010 World Health Organization classification system: Well-differentiated neuroendocrine tumor (NET; Grade 1 and Grade 2, Ki-67 $\leq 20 \%$ and/or mitotic count $\leq 20$ per 10 high-power fields), poorly differentiated neuroendocrine carcinoma (NEC; Grade 3, Ki-67 $>20 \%$ and/or mitotic count $>20$ per 10 high-power fields) and mixed adenoendocrine carcinoma (MANEC) (3).

Different pathological types vary greatly with regards to biological behavior and prognosis. Generally, NET tends to be biologically indolent and has a favorable prognosis, whereas NEC is an aggressive tumor type associated with poor patient survival rates (1). However, at present, the characteristics of MANEC remain poorly elucidated due to the histological complexity of this tumor type. MANEC refers to a composite tumor characterized by coexisting glandular and neuroendocrine elements, with each accounting for $>30 \%$ of 
the lesion. Although MANEC is not frequently encountered, the coexistence of two distinct histological components in the same tumor provokes interest from clinicians and researchers. Additionally, controversies exist regarding the pathogenesis of biphasic morphology and the therapeutic protocols for this particular subtype (4).

The extrahepatic biliary tract (EHBT) arises from outside the liver and extends to the ampulla. It has a complex anatomical position, as it is surrounded by diverse structures, including the pancreas, duodenum, portal vein, hepatic artery and autonomic nerve fibers (5). Cholangiocarcinoma (CCA) is the most common type of tumor in the EHBT, which accounts for $>80 \%$ of cases (6). NEN at this location is rare, even more so MANEC. However, cases of biliary NENs have been increasingly described in the medical literature, presumably due to a true increase in incidence or advances in diagnostic tools $(4,7,8)$.

The data suggests that NEN in the EHBT has a tendency to mimic conventional CCA in terms of biological heterogeneity, presenting substantial challenges for clinical management and prognostic stratification (8). Several attempts have been made to clarify the clinical settings of this unusual disease. However, prior studies only focused on a single pathological type (mainly NET or NEC) (8-10). Additionally, currently, the prognostic factors of NEN in the EHBT have not been investigated (11).

In the present report, a MANEC in the distal common bile duct (CBD) in a 64-year-old Chinese female patient was described. Additionally, previous cases of NENs in the EHBT were collected from the medical literature and reviewed to provide centralized clinical data and to identify factors affecting the survival outcome of patients with NEN.

\section{Case report}

A 64-year-old Chinese woman presented to a local hospital (the first people's hospital of Fuyang, Hangzhou, China) in July 2015 with complaints of abdominal pain and obstructive jaundice. The patient had no fever, nausea or vomiting. The patient had undergone cholecystectomy for gallstones 2 years earlier. There was no history of trauma, bronchospasm, peptic ulcers or cancer. Imaging examinations suggested a malignant structure at the distal CBD, with significant upstream tract dilation. Although surgery was offered, the patient refused and three biliary stents were placed instead. Later, the patient's discomfort gradually subsided. The patient was discharged after a total of 10 days in the local hospital. However, the patient complained again of abdominal pain 5 months later (in December 2015) and was referred to the First Affiliated Hospital, School of Medicine, Zhejiang University (Hangzhou, China).

On admission (December 2015), the patient had no fever, jaundice, diarrhea, hypotension or flushing. Physical examination revealed slight upper abdominal tenderness without rebound tenderness or guarding. Laboratory work-ups highlighted elevated levels of serum carcinoembryonic antigen (31.3 ng/ml, normal 0-5 ng/ml) and carbohydrate antigen 19-9 (40.4 U/ml, normal 0-35 U/ml). Liver function tests were within normal limits. The chest X-ray was negative. An abdominal computed tomography scan revealed that the distal CBD had thickened and moderately enhanced duct walls. Magnetic resonance cholangiopancreatography indicated that the common hepatic duct, proximal and middle CBD, and main pancreatic duct were markedly dilated (Fig. 1A). Endoscopic retrograde cholangiopancreatography (ERCP) indicated severe stenosis at the distal CBD (Fig. 1B), and the biliary stents were retrieved. Biliary duct brush cytology at the time of ERCP revealed a small cluster of atypical cells. The patient was tentatively diagnosed with distal CCA, and a standard Whipple pancreaticoduodenectomy was scheduled. Intraoperatively, the patient was found to have enlarged lymph nodes in the hepatic hilum, which were removed. This procedure was considered curative since the intraoperative frozen section revealed that the resected margins were free of atypical cells. Macroscopically, a grayish, solid tumor surrounded, infiltrated and extended along the distal CBD wall. Subsequent to being resected and flattened, the tumor measured $4.5 \times 3.0 \mathrm{~cm}$ in size.

The $4 \mu \mathrm{m}$-thick surgical specimens were fixed with $10 \%$ neutral formaldehyde solution at room temperature, paraffin embedded, and were then subject to detailed histopathological analysis combined with immunohistochemical (IHC) staining. The immunostaining was performed according to the standard protocol of the Department of Pathology, First Affiliated Hospital, School of Medicine, Zhejiang University (Hangzhou, China). Briefly, the specimens were cut into 4- $\mu$ m thick sections, deparaffinized, and rehydrated and $1.5 \%$ hydrogen peroxide in methanol was used for the blockage of endogenous peroxidase at room temperature. Then, the sections were washed with distilled water, and immersed in the heated EGTA solution (pH 9.0) for $20 \mathrm{~min}$. After cooling down, the sections were washed with phosphate-buffered saline (PBS; pH 7.2-7.6, three times). Subsequently, The tissue sections were incubated with a panel of 12 primary antibodies overnight at $4^{\circ} \mathrm{C}$, including caudal type homeobox 2 (CDX2; ZA-0520 EP25; 1:100 dilution), mucin (MUC) 1 (ZA-0656 EP85; 1:100 dilution), MUC2A (MRQ-18; 1:200 dilution), cytokeratin 19 (CK19; K19.2; 1:200 dilution), MUC5AC (ZA-0664; 1:100 dilution), mammaglobin (MMG; ZM-0388; 1:80 dilution), cluster of differentiation 56 (CD56; 123C3.D5; 1:80 dilution), synaptophysin (ZA-0506; 1:200 dilution), chromogranin-A (LK2H101-PHE5; 1:100 dilution), Ki-67 (ZM-0167; 1:1,000 dilution), thyroid transcription factor-1 (TTF-1; ZM-0270; 1:200 dilution) and gross cystic disease fluid protein-15 (GCDFP-15; 23A3; 1:100 dilution). CD56, chromogranin-A, CK19 and GCDFP-15 antibodies were obtained from Shanghai Long Island Biotec. Co., Ltd. (Shanghai, China), and the remaining antibodies were obtained from OriGene Technologies, Inc. (Rockville, MD, USA). The sections were subsequently washed with PBS (three times), and anti-mice/rabbit enzyme-labeled secondary antibodies [PV-8000 (IB000086), 1:200 dilution; provided by the Zhong Shan Golden Bridge Biological Technology Inc., Beijing, China; EnVision detection system] were then applied at room temperature for $15 \mathrm{~min}$. The slides were rinsed in PBS again (three times), and treated with diaminobenzidine (DAB; 1:50) for $5 \mathrm{~min}$, rinsed in distilled water, and finally counterstained with hematoxylin according to a standard protocol. Leica DM2500 optical microscopes were used and Ki-67 scoring was performed as described by Adsay (12).

The results revealed a collision tumor composed of poorly differentiated adenocarcinoma and NEC (Fig. 2). The adenocarcinoma component was poorly differentiated, exhibited an 

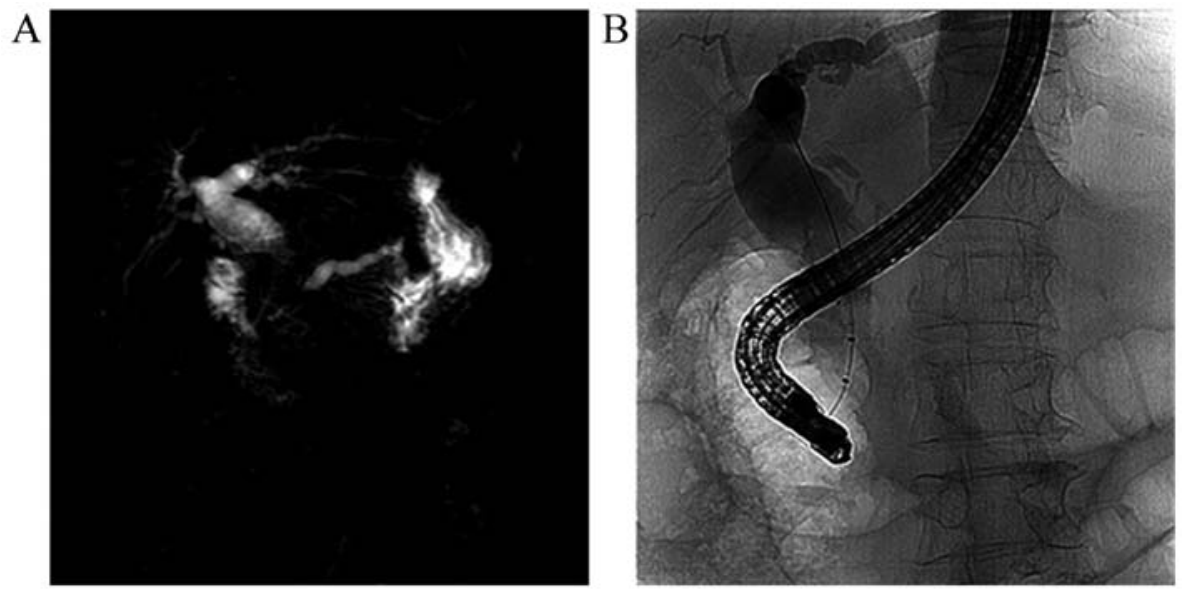

Figure 1. (A) Magnetic resonance cholangiopancreatography and (B) endoscopic retrograde cholangiopancreatography. The images show severe stenosis at the distal common bile duct (white arrows). Marked dilations of the proximal biliary tract and main pancreatic duct were observed.
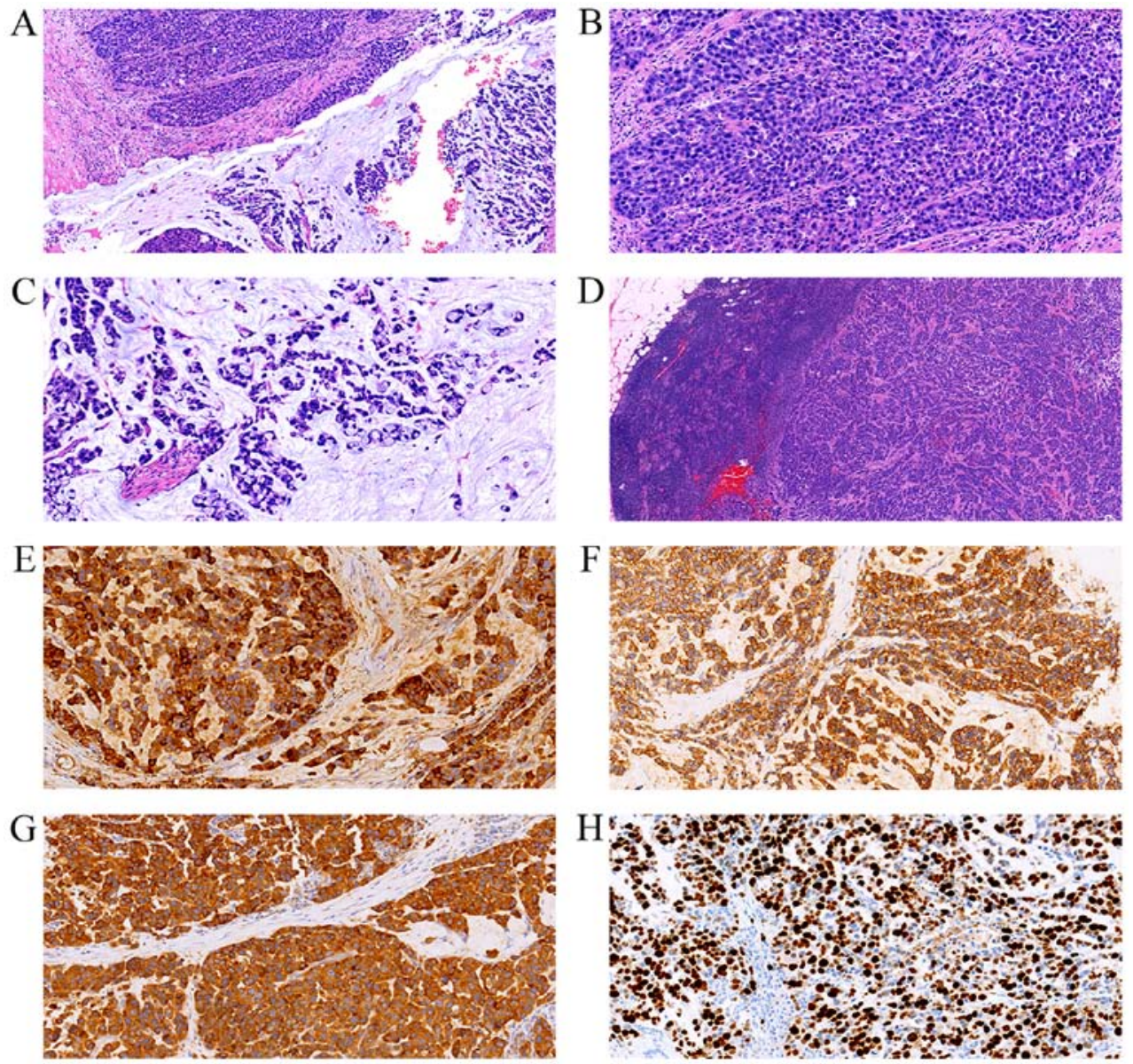

Figure 2. Pathological analysis of the surgical specimen. (A) Tumor was composed of adenocarcinoma and neuroendocrine components, with each occupying $>30 \%$ of the lesion. The two components were arranged in a clearly separated pattern (H\&E; magnification, $x 100)$. (B) Neuroendocrine component was formed by small tumor cells with scant cytoplasm and hyperchromatic nuclei (H\&E; magnification, x200). (C) Mucin pool and signet ring-like cell clusters were noted in the adenocarcinoma component (H\&E; magnification, x200). (D) Metastatic lesions in the lymph nodes were predominantly neuroendocrine carcinoma (H\&E; magnification, $\mathrm{x} 40$ ). Immunohistochemical staining revealed that the neuroendocrine component was strongly positive for (E) chromogranin A (magnification, x200), (F) cluster of differentiation 56 (magnification, x200) and (G) synaptophysin (magnification, x200), with a (H) Ki-67 labeling index $>50 \%$ (magnification, x200). H\&E, hematoxylin and eosin.

intestinal phenotype, and accounted for more than $30 \%$ of the tumor. Mucin pool and signet ring-like cells were observed. Tumor cells were stained for CDX2, MUC2A, CK19 and focally MUC5AC. The NEC component accounted for $\sim 60 \%$ of the tumor, and was characterized by small tumor cells with scant cytoplasm, hyperchromatic nuclei and inconspicuous nucleoli. These tumor cells were arranged in a nesting pattern and they strongly expressed CD56, synaptophysin and chromogranin-A, 
with a Ki-67 labeling index $>50 \%$. MUC1, GCDFP-15, TTF-1 and MMG were negative. The angiolymphatic invasion were predominantly NEC. In view of these findings, the tumor was definitively diagnosed as a MANEC in the distal CBD.

Following surgery, the patient recovered and was discharged from the hospital after 2 weeks. However, repeated imaging studies postoperatively over 8 months revealed multiple intrahepatic and pulmonary metastases. The patient succumbed to disease 12 months after the surgery.

\section{Discussion}

MANECs predominately occur in the colon, appendix and stomach, where neuroendocrine cells are diffusely distributed (4). MANECs arising from the EHBT are extremely rare, with a total of eight cases reported in the medical literature since the category was introduced in 2010 (13-20). The histogenesis of biliary MANEC remains under debate due to the scarcity of enterochromaffin (Kultchisky) cells in the normal bile duct (21). To explain this issue, the following theories have been formulated.

As mentioned in the presented case and other studies, intestinal metaplasia is a frequent and well-documented event in MANEC $(22,23)$. A case of biliary NEC has been described in which histopathological analysis revealed concurrent dysplasia with intestinal and neuroendocrine differentiations in the biliary tracts within and adjacent to the invasive NEC (9). Given the close histopathological associations, intestinal metaplasia of the biliary epithelium may be involved in the development of MANEC following a sequence of metaplasia-dysplasia-carcinoma.

Using surgical specimens of biliary MANECs, the expression levels of Notch1, Jagged1 and hes family bHLH transcription factor 1 (Hes1) have been demonstrated to be constant in the adenocarcinoma component, but decreased or absent in the neuroendocrine component (24). Additionally, disruption of the Notch1-Hes1 signaling axis significantly increases the expression profiles of neuroendocrine protein markers in a cultured CCA cell line (24). Collectively, this evidence suggests that biliary MANEC may be associated with the transdifferentiation of adenocarcinoma (24). A hypothesis was proposed that it may result from proliferation of a common precursor stem cell, which is capable of divergent differentiation. This hypothesis was supported by the observation that prominin 1, a biomarker of cancer stem cells, is expressed in $63.6 \%$ of cases of digestive MANECs (25). Evidence obtained from next-generation sequencing in nonbiliary digestive MANECs also suggests a monoclonal origin of the two histological components (26).

Histopathologically, the glandular component of MANEC is generally detected at the tumor surface, while the neuroendocrine component is located in the deep stroma; the latter is typically responsible for tumor invasiveness (23). The two tumoral phases of MANEC may be arranged in either clearly separated (collision) or tightly mingled (combined) patterns; much less frequently, the tumor cells exhibit a mixed adenocarcinomatous-neuroendocrine (amphicrine) phenotype (27).

NEN in the EHBT represents an uncommon disease accounting for $0.1-0.2 \%$ of all gastroenteropancreatic NENs. However, patients with this unusual entity exhibit a wide spectrum of oncology outcomes, ranging from curative following tumor excision to a poor prognosis even following multidisciplinary treatment $(10,28)$. Accumulating clinical data suggests that the prognostic heterogeneity may be associated with pathological classification $(10,11,23,26)$. However, a straightforward comparison among the three pathological types is currently lacking due to the rarity of the disease. To gain an improved understanding of NEN in the EHBT by incorporating all pathological types and to identify prognostic predictors, a literature review of pertinent publications in the English literature was conducted.

PubMed (www.ncbi.nlm.nih.gov/pubmed) was searched for English language studies that described NEN in the EHBT between 2010 and 2018 using medical terms, including 'carcinoid', 'mixed adenoendocrine carcinoma', 'neuroendocrine neoplasm', 'neuroendocrine tumor', 'neuroendocrine carcinoma', 'biliary duct' and 'bile duct'. In the present study, 2010 was selected as the starting year since this is when the latest classification system of NEN was introduced. Cases of NENs located in the gallbladder, cystic duct and the ampulla of Vater were excluded. Aggregated data for patients with biliary NEN from series studies were also excluded where patient-level information was not available. The search identified 37 patients with NEN in the EHBT since 2010. Eventually, a total of 38 cases, including the present case, were analyzed (Table I) $(9,10,13-20,28-53)$. Clinical characteristics and survival outcomes among different pathological types were compared, and they are summarized in Table II.

Preliminary Shapiro-Wilk tests demonstrated the skewed distributions of quantitative variables, which were therefore expressed as median and interquartile range (IQR) and compared by Kruskal-Wallis tests. $\chi^{2}$ tests were used for categorical variables. The survival analysis was conducted by the Kaplan-Meier method, and the log rank test was used for comparisons among groups. Notably, Cox proportional hazards regression analysis was not performed due to the limited data for survival analysis $(n=31)$. Statistical analysis was performed using IBM SPSS Statistics v19.0 software (IBM Corp., Armonk, NY, USA). P $<0.05$ was considered to indicate a statistically significant difference.

Of the 38 cases of NEN in the EHBT (including the present case), the majority were NET $(n=22,57.9 \%)$. Biliary NECs and MANECs were less common, with a total of seven (18.4\%) and nine $(23.7 \%)$ cases identified, respectively. The median age of patients at diagnosis was 62 years (IQR 43.5-75.0), and males were slightly predominant $(\mathrm{n}=23,60.5 \%)$. The tumor size ranged between 1.0 and $6.0 \mathrm{~cm}$, with a median of $2.4 \mathrm{~cm}$ (IQR 1.9-3.0). The involvement of the perihilar biliary tract was noted in 15 cases. Patients with biliary MANEC had a median age of 75 years at diagnosis, which was comparable to that of patients with NEC (median 75 years) but significantly higher than that of patients with NET (median 45 years; $\mathrm{P}<0.001$ ). Males appeared to be predominant in the MANEC and NET groups, with a male:female ratio of 7:2 and 13:9, respectively, while no gender discrepancy was noted in NEC (3:4). Compared with NET and MANEC, NEC tended to exhibit a larger tumor size and to be more frequently associated with the involvement of the perihilar biliary duct; however, these differences did not reach statistical significances $(\mathrm{P}=0.369$ and $\mathrm{P}=0.41$, respectively). 


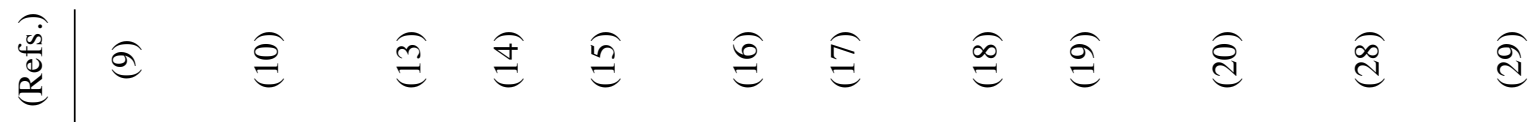

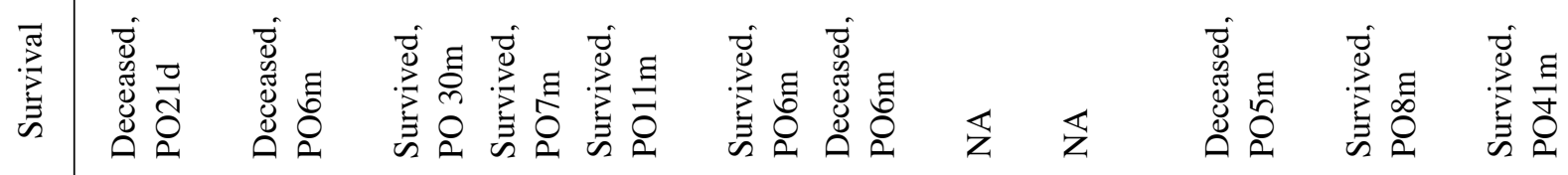

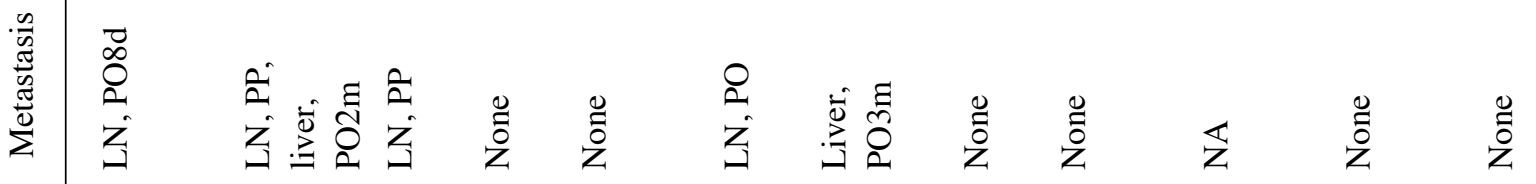

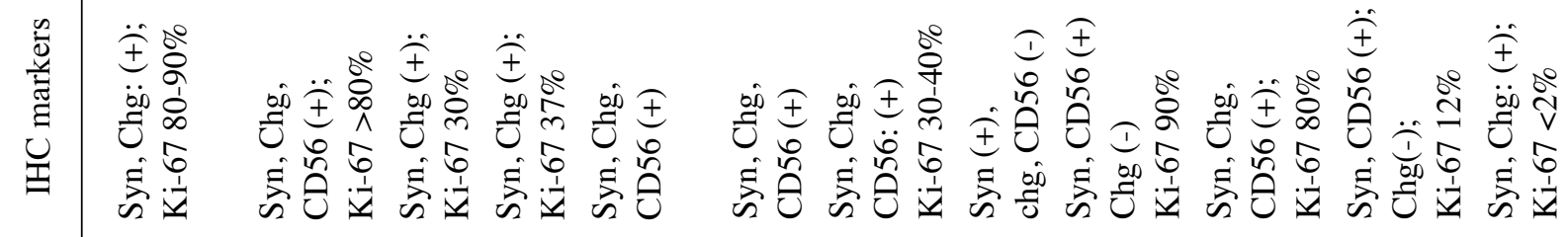

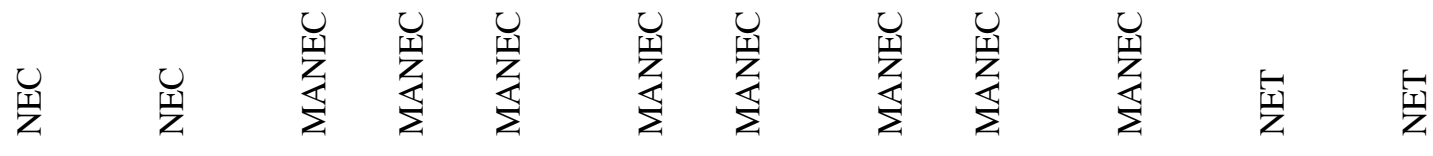

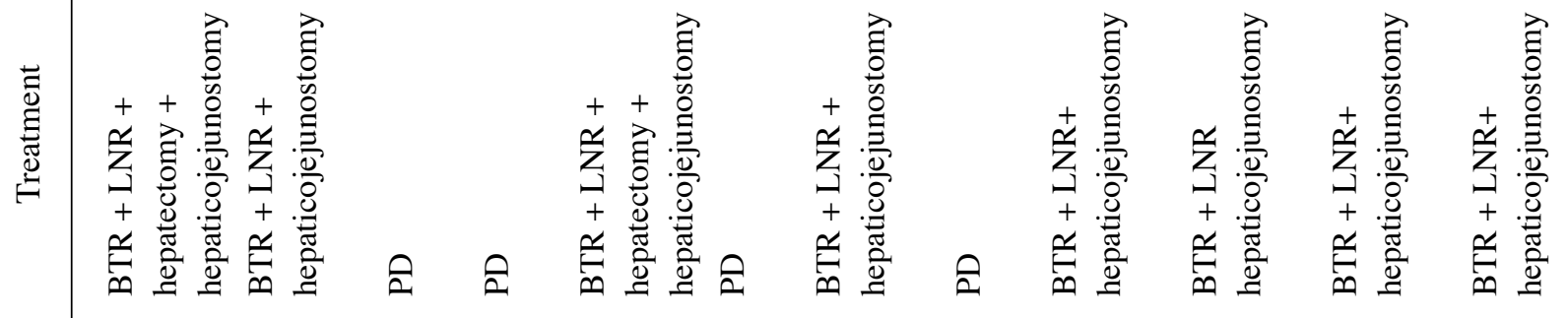

总危

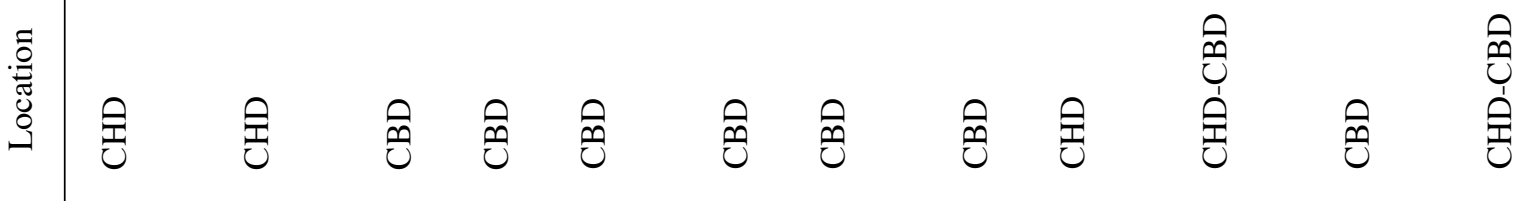

产

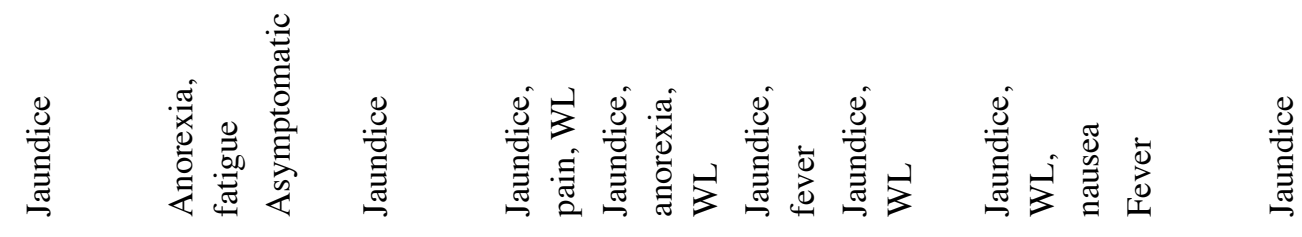

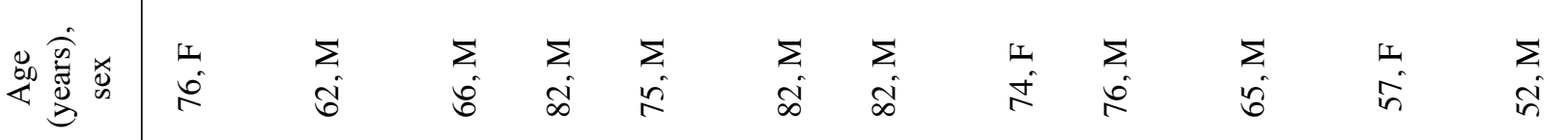

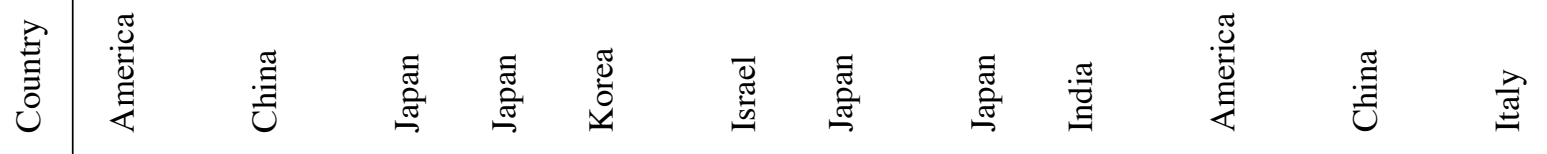

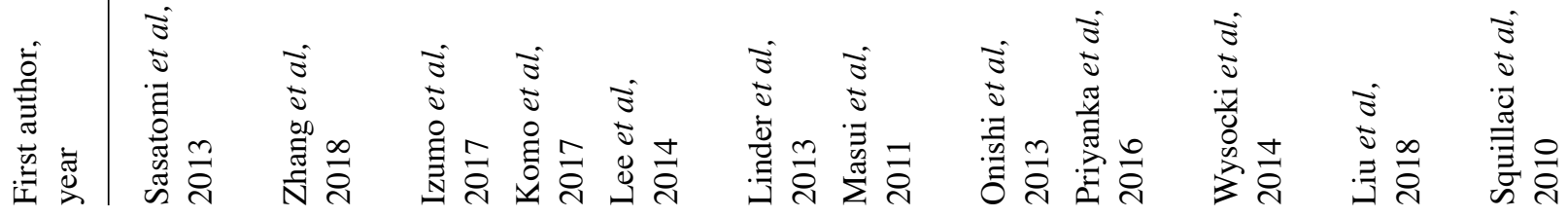




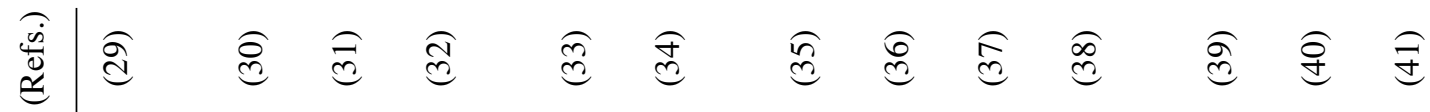

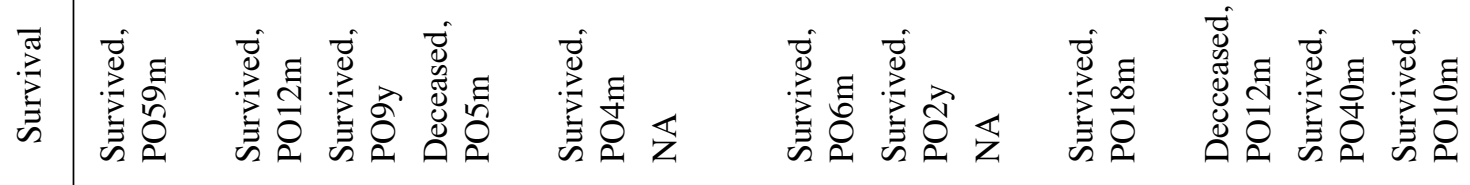

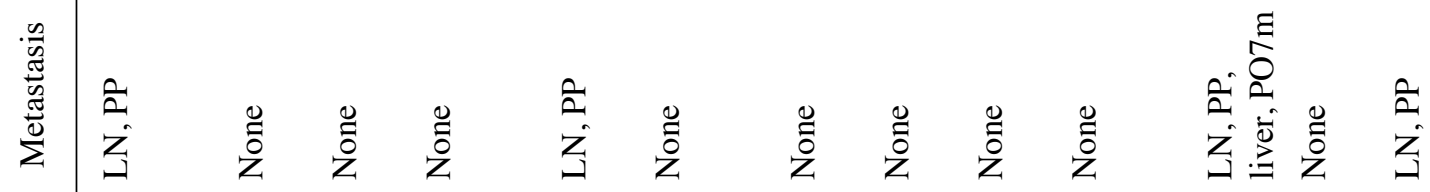

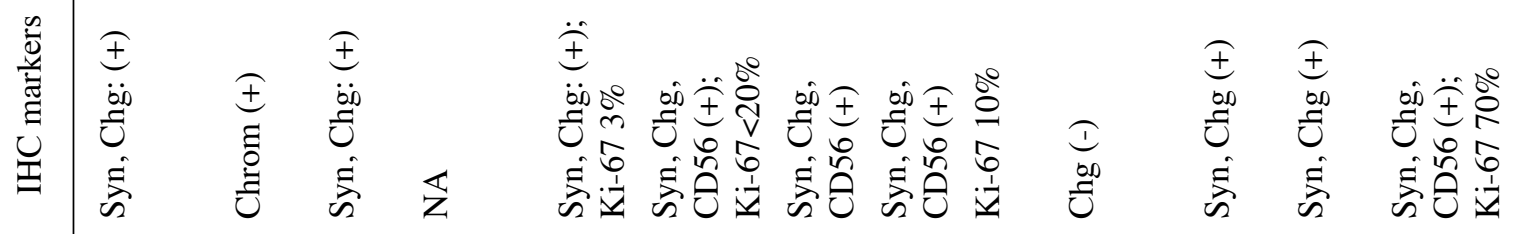

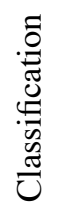

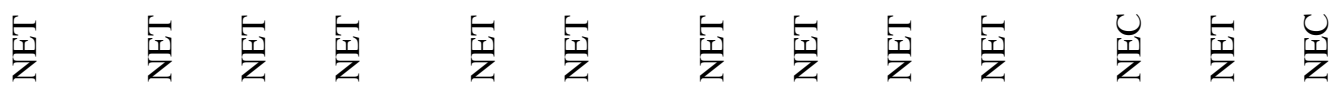

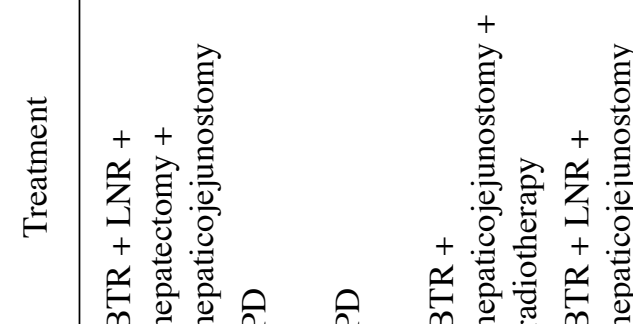

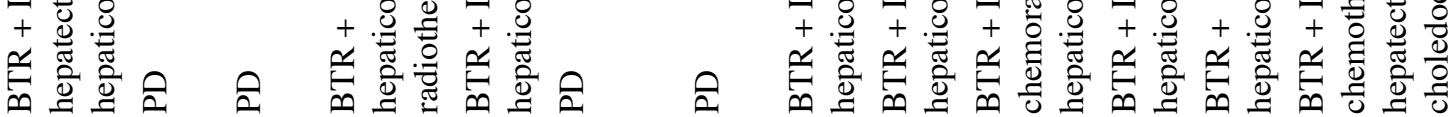

总爱

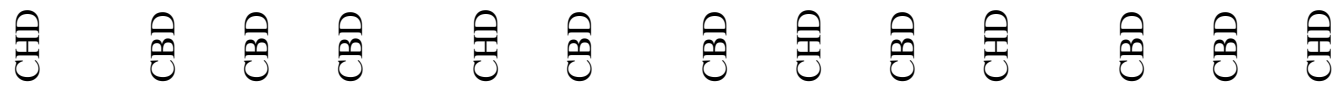

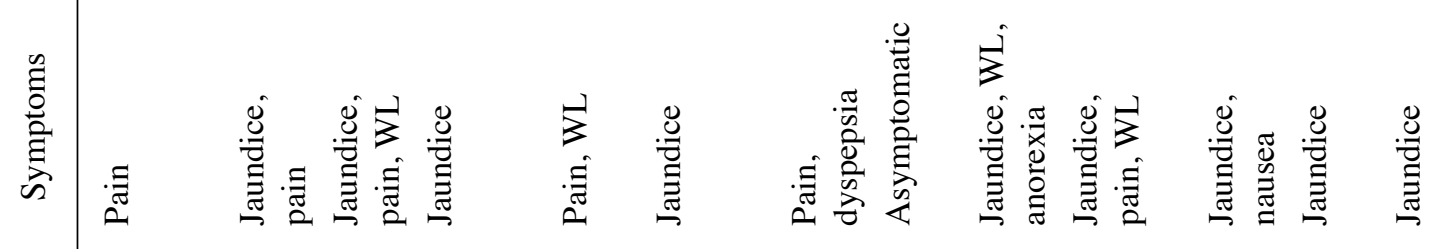

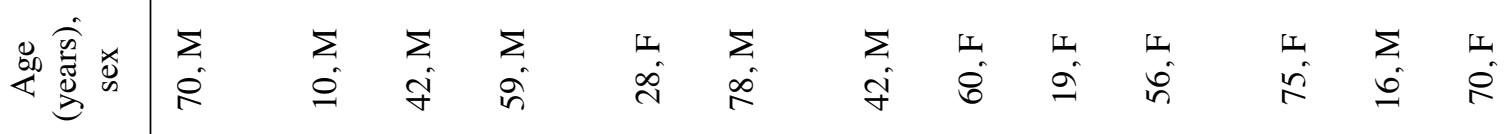

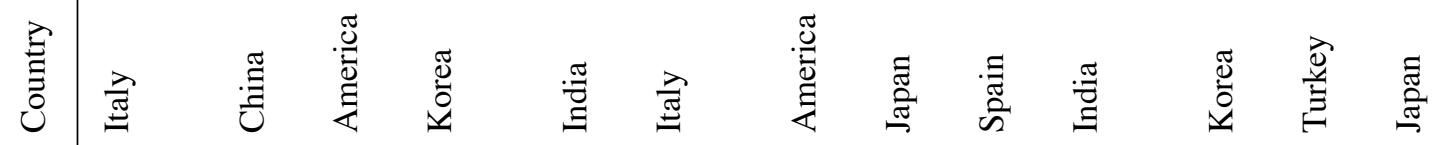




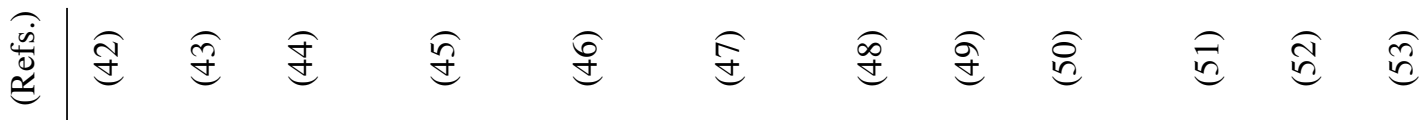

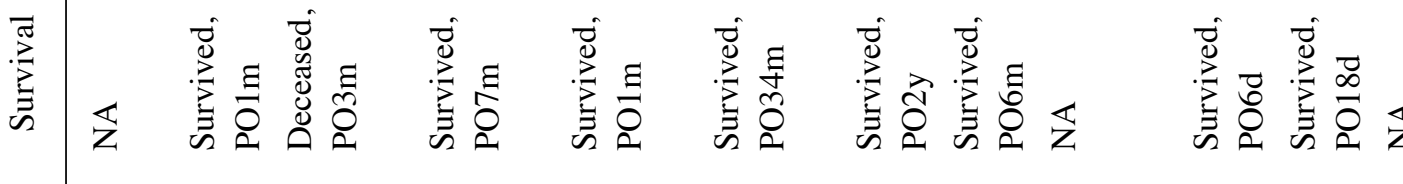

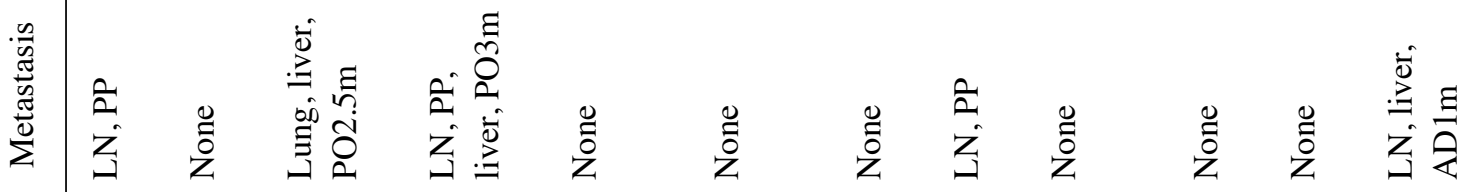

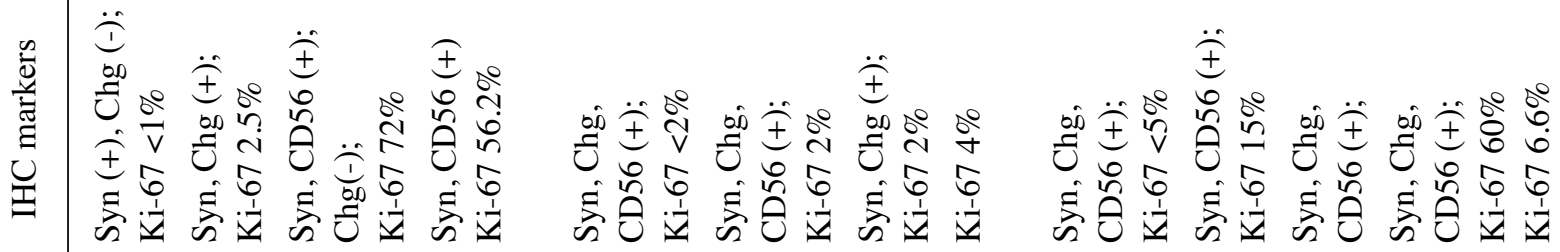

童

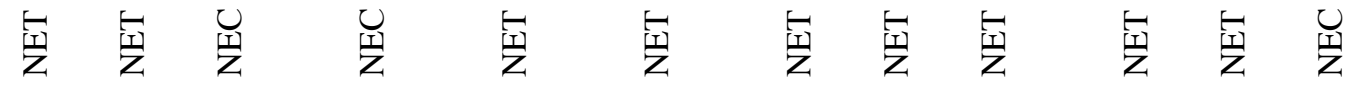

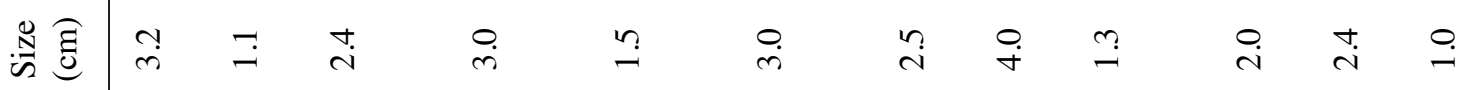

害

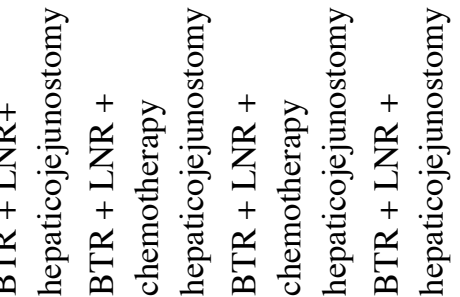

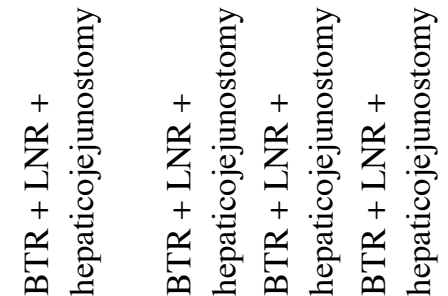

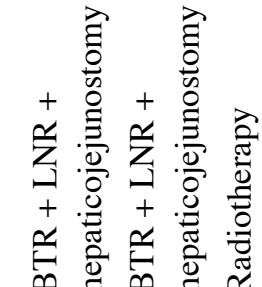

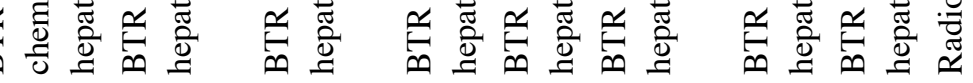

ชิ

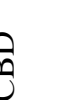

悹

:

总 总

递

莺

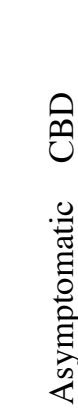

ชิ 0ิن

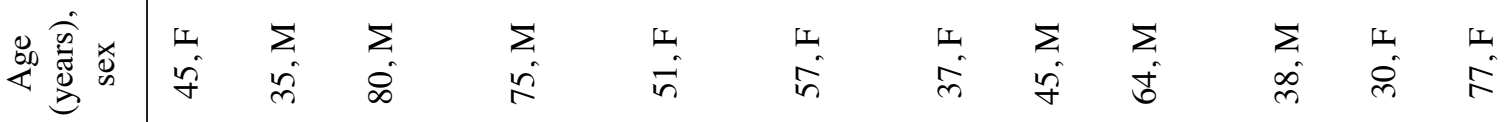

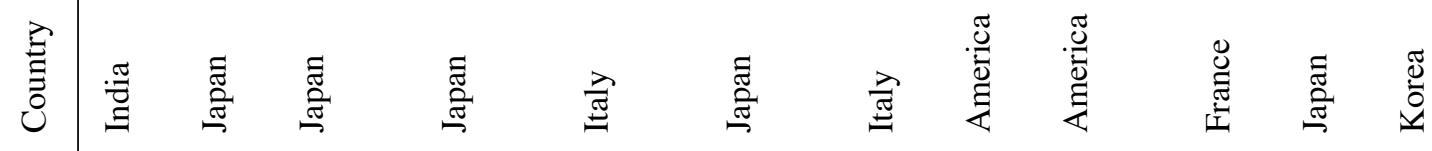

$\overrightarrow{2} \approx \overrightarrow{0}$

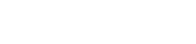


Fairly well recognized gastroenteropancreatic NENs were characterized by the capability to produce bioactive substances that cause characteristic hormonal symptoms. It has been estimated that the carcinoid syndrome (including flushing, hypotension, diarrhea and bronchospasm) is presented in $\sim 1 / 3$ patients with small intestinal NETs; $40-55 \%$ of pancreatic NETs may be classified as functional tumors (54). Nonetheless, in this literature review, symptoms caused by biliary NEN were mostly due to the mass effect; functional symptoms tended to be absent in all pathological subtypes. The most common symptom of biliary NEN was jaundice (27 patients, $71.1 \%$ ), followed by fever (10 patients, $26.3 \%$ ), abdominal pain (9 patients, 23.7\%), anorexia/nausea dyspepsia/fatigue/discomfort (8 patients, $21.1 \%$ ) and weight loss (two patients, 5.3\%); 4 patients were asymptomatic (10.5\%) at diagnosis. In parallel with the lack of symptoms, biliary NEN was rarely identified to be associated with abnormal hormone levels. Biochemical tests have frequently highlighted the elevated levels of serum carcinoembryonic antigen and carbohydrate antigen 19-9, as described in $3 / 21$ cases (14.3\%) and $15 / 36$ cases (41.7\%), respectively (data not shown). Although efforts have been made, the measurement of serum hormone levels was not technically feasible for the presented case. In the majority of cases reported in the literature, the hormone levels are within the normal range, contributing to the high rate of misdiagnosis of these cases as CCA (8-11,13-20,28-53).

Imaging results for biliary NEN generally overlap with those of CCA, leading to a high rate of misdiagnosis. Generally, a computed tomography scan depicts a hypodense, well-vascularized and heterogeneously enhanced lesion. Upstream bile-duct dilation and lymph-node enlargement are common findings. Biliary NENs on magnetic resonance imaging mostly appear as nodular (45\%) and intraductal-growing $(45 \%)$ shapes and less frequently as periductal-infiltrating (9\%) type (55). In positron emission tomography, NEN usually demonstrates high glucose metabolism, particularly for poorly differentiated NEC $(44,56)$.

Due to the unspecific clinical and imaging characteristics, an accurate preoperative diagnosis of biliary NEN is extremely difficult. In the majority of cases, histopathological analysis completed by IHC investigations of surgically resected specimens is required to achieve a definitive diagnosis. Macroscopic examinations of tumors usually reveal a nodular, infiltrating or polypoid mass. Histopathologically, tumors tend to exhibit cord, nest or trabeculae growth patterns. Perineural and lymphovascular invasions have been frequently observed. To confirm the neuroendocrine phenotype and the grade of the NENs, IHC investigation is required. Among the commonly used markers, synaptophysin and chromogranin are two of the most reliable neuroendocrine markers (57). NEN usually stains diffusely for synaptophysin due to the presence of small clear vesicles in tumor cells and for chromogranin due to large neurosecretory granules. Neuron-specific enolase and CD56 have been identified to exhibit a lower specificity. The Ki-67 staining index and mitotic count are crucial for tumor grading, as defined in the classification systems (3). Specifically, the $\mathrm{Ki}-67$ index is generally more accurate and reproducible when compared with mitotic count.

The standardized management protocols for NEN in the EHBT have not been well developed due to limited experience 
Table II. Comparison of NET, NEC and MANEC in the extrahepatic biliary tract.

\begin{tabular}{|c|c|c|c|c|c|}
\hline Variable & NEN & NET & NEC & MANEC & P-value \\
\hline No. of cases & 38 & 22 & 7 & 9 & \\
\hline Male/female & $23 / 15$ & $13 / 9$ & $3 / 4$ & $7 / 2$ & \\
\hline Age, years (IQR) & $62.0(43.5-75.0)$ & $45.0(36.0-58.0)$ & $75.0(70.0-77.0)$ & $75.0(65.5-82.0)$ & $<0.001$ \\
\hline \multicolumn{6}{|l|}{ Symptoms, no. } \\
\hline Jaundice & 27 & 14 & 7 & 7 & \\
\hline Abdominal pain & 9 & 7 & 1 & 2 & \\
\hline Weight loss & 2 & 1 & 0 & 1 & \\
\hline Fever & 10 & 6 & 0 & 4 & \\
\hline Asymptomatic & 4 & 3 & 0 & 1 & \\
\hline $\begin{array}{l}\text { Anorexia/nausea/dyspepsia/fatigue } \\
\text { /discomfort }\end{array}$ & 8 & 3 & 2 & 3 & \\
\hline Tumor size, cm (IQR) & $2.4(1.9-3.0)$ & $2.0(1.8-3.0)$ & $2.7(2.0-3.0)$ & $2.0(1.9-4.3)$ & 0.369 \\
\hline Tumor location & & & & & $0.41^{\mathrm{c}}$ \\
\hline CHD involved & 15 & 9 & 4 & 2 & \\
\hline Only CBD involved & 23 & 13 & 3 & 7 & \\
\hline Mean follow-up time, months (IQR) & $7.0(5-24)$ & $10.0(3.3-35.5)$ & $6.5(2.5-10.5)$ & $7.0(6.0-12.0)$ & 0.611 \\
\hline Mortality, no. (\%) & $8(26 \%)$ & $1(5 \%)$ & $4(66.7 \%)$ & $3(42.9 \%)$ & \\
\hline Recurrent events, no. (\%) & $6(19.4 \%)$ & 0 & $5(71.4 \%)$ & $2(28.6 \%)$ & \\
\hline Survival, months & & & & & $0.006^{\mathrm{b}}$ \\
\hline Mean & 72.2 & 100 & 7.7 & 16.6 & \\
\hline Median & - & - & 6 & 12 & \\
\hline
\end{tabular}

CBD, common bile duct; CHD, common hepatic duct; IQR, interquartile range; MANEC, mixed adenoendocrine carcinoma; NEN, neuroendocrine neoplasm; NET, neuroendocrine tumor; NEC, neuroendocrine carcinoma. 'Obtained from comparisons among the three pathological types of biliary NEN; ' log-rank test; 'Obtained from $\chi^{2}$ test, a statistical difference referring to 'CHD involved' and 'only CBD involved'.

with this uncommon disease. Radical surgery represents a potentially curative option, and it has been recommended for cases of all pathological types where imaging examinations suggest that complete resection is feasible. The surgical procedures heavily depend on the primary tumor sites. Of the cases considered in the present study, pancreaticoduodenectomy was performed in 10 cases, mainly for tumors located in the distal CBD. Surgical resections for perihilar NENs involved bile-duct excision, lymph-node dissection, and Roux-en-Y hepaticojejunostomy or hepaticoduodenostomy, with or without hepatic lobectomy, as performed in 27 cases. In $6 / 31$ cases $(19.4 \%$ ), the tumor recurred following surgical resection; four were NECs and two were MANECs. Notably, no tumor recurrence was noted in cases of biliary NETs over a median follow-up period of 10 months.

Surgical resection usually provides a curative chance for patients with biliary NET. Adjuvant therapies are not required for completely resected well-differentiated NET (58).

For the cases of NECs in the EHBT considered in this study, systematic chemotherapy was frequently employed (3/7 cases, $42.9 \%)$ and aimed to improve resectability or to control tumor progression. There is no standard chemotherapy regimen established for biliary NEC. The most common chemotherapy regime consists of a combination of cisplatin and etoposide, borrowing from treatment experiences with pulmonary NEC. A higher chemotherapy response rate for advanced NEC may be expected when the tumor presents with a higher Ki-67 proliferative index (>55\%) (59).

The chemotherapy regimen selection for MANEC remains a large clinical dilemma, since it is complicated by the mixture of distinctive malignant histologies. Without clear consensus based on evidence, attempts at adjuvant therapies have been seldom made in patients with biliary MANEC. However, as recurrent events were noted in $2 / 9$ patients $(22.2 \%)$, adjuvant chemotherapy may be justified. Further studies are required to tailor chemotherapy strategies, and to determine which component to target to obtain the best therapeutic benefits.

During a median follow-up period of 7 months, a total of eight patients succumbed to disease, of which one, four and three were diagnosed with NET (12.5\%), NEC (50\%) and MANEC $(37.5 \%)$, respectively. To identify parameters useful for prognostic stratification, Kaplan-Meier analysis and a log-rank test were performed for 31 patients with survival data.

As anticipated, the survival outcome of patients with NEN in the EHBT varied significantly by pathological type. The median overall survival for patients diagnosed with NET, NEC and MANEC was 100 (data not shown), 7.7, and 16.6 months, respectively $(\mathrm{P}<0.001$, Fig. $3 \mathrm{~A}$; the $\mathrm{X}$-axis was shortened regarding the great difference of median overall patient survival among three pathological types). Additionally, old age and tumor recurrence were identified to negatively affect clinical outcomes (Fig. 3B and C). Patients aged 

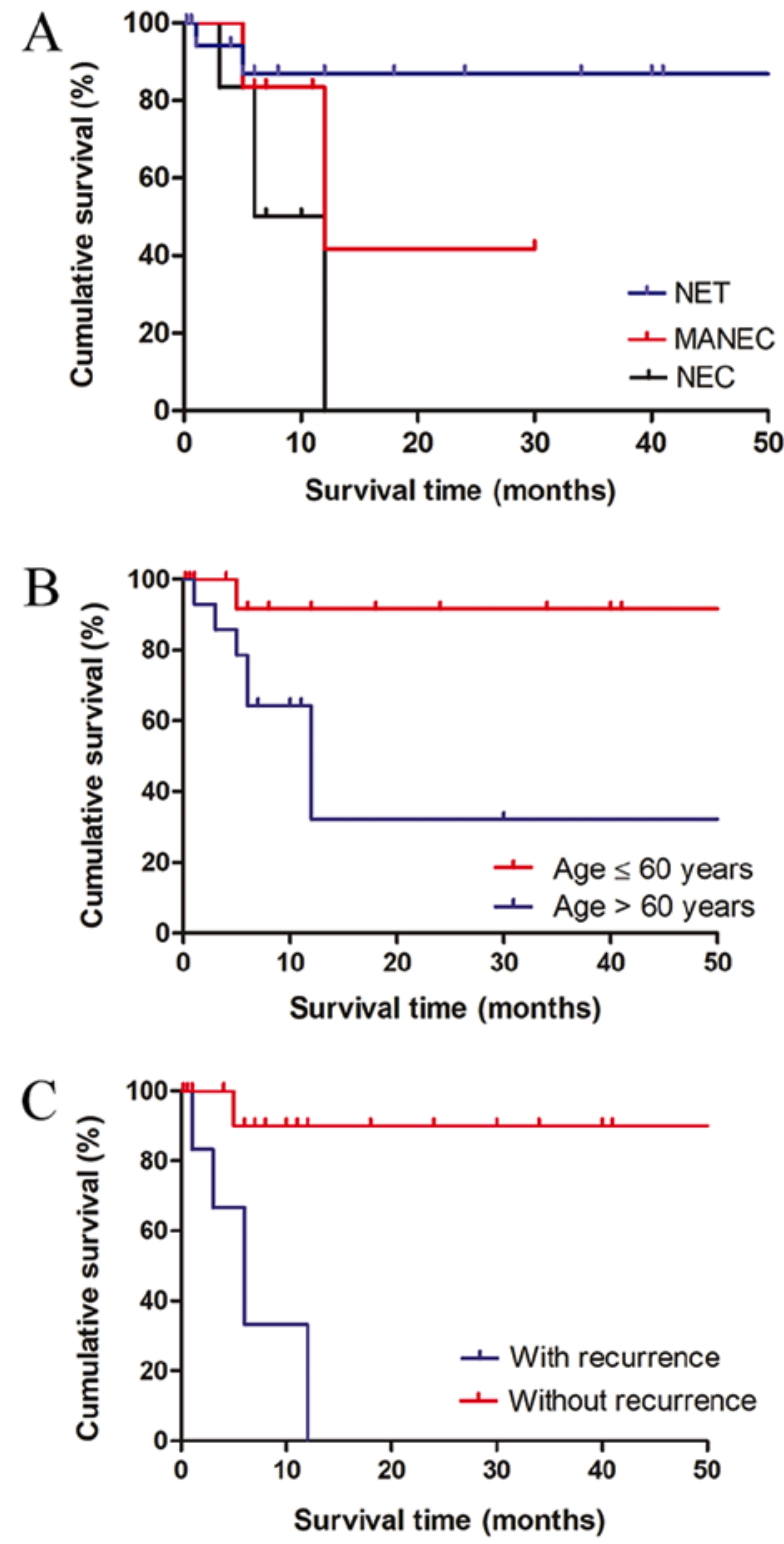

Figure 3. Kaplan-Meier survival curves of patients with neuroendocrine neoplasm in the extrahepatic biliary tract. (A) NEC was associated with the worst prognosis, followed by MANEC and NET $(\mathrm{P}<0.001)$. (B) Patients aged $>60$ years exhibited a worse survival rate compared with those who were $\leq 60$ years old $(\mathrm{P}=0.033)$. (C) Tumor recurrence was associated with a decreased survival rate $(\mathrm{P}<0.001)$. MANEC, mixed adenoendocrine carcinoma; NEC, neuroendocrine carcinoma; NET, neuroendocrine tumor.

$>60$ years exhibited significantly worse survival than those who were $\leq 60$ years old $(\mathrm{P}=0.033)$. A significantly shorter overall survival was observed in patients with postoperative tumor recurrence than those without $(\mathrm{P}<0.001)$. Sex, tumor size and location were identified to not be associated with survival outcomes (data not shown).

The findings of the present study were consistent with the results from prior studies that investigated NENs from other primary sites, and once again, they highlighted the pivotal role of histological classification in predicting tumor biological behavior and clinical outcome. A national surveillance, epidemiology and end results (SEER) survey of appendiceal MANECs demonstrated that MANEC is associated with a significantly worse prognosis than NET, with a median overall survival of 6.5 and 39.4 years $(\mathrm{P}<0.001)$, respectively $(60)$. In the same study, patients with MANEC were revealed to be older compared with patients with NET (58 vs. 40 years; $\mathrm{P}<0.001$ ) (60). However, the discrepancy between MANEC and NEC prognoses may vary by primary sites. Similar to the findings of the present study, which suggested that the prognosis of biliary MANEC was superior to that of NEC, patients with gastric MANEC exhibit an improved survival rate compared with those with pure NEC (61). Conversely, in a previous study no significant survival differences were identified in patients with colorectal MANEC and NEC (62). Furthermore, the negative effect of older age on patient survival has also been documented. In line with the results of the present study, a SEER survey comprising 35,618 patients with gastrointestinal NEN demonstrated that age is a strong predictor of survival duration; those aged $>60$ years exhibit the worst outcome at all disease stages $(\mathrm{P}<0.001)(7)$.

Since the present analysis was mostly based on case reports in the literature, a reporting bias may potentially exclude the publication of similar cases and thus underestimate the true incidences of NET and NEC in the EHBT. A previous collection of cases of NETs in the EHBT from 1959 to 2012 yielded a total of 150 cases in the medical literature (8). Similarly, our previous study collected a total of 21 cases of biliary NECs from the literature (10). These studies, however, only focused on a single pathological subtype. Distinctively, the present survey included all pathological types from prior publications, and centralized data is therefore provided in brief. Nonetheless, the limited number of cases could potentially weaken the statistical power, and it hampered the Cox proportional hazards model analysis for independent prognostic factors. However, this is inevitable, as the disease itself is uncommon. Additionally, the heterogeneity of management protocols among the centers of different authors would constitute a prominent confounding bias that affects patient prognosis. The absence of consistent descriptions among these case reports restricted the extended analysis for other potential parameters, including the Ki-67 index, tumor stage and surgery-associated variables. To explore their prognostic relevance, further studies based on a large series of complete data are required.

In conclusion, NEN infrequently occurs in the EHBT, with NET representing the predominant type. NEN in the EHBT is challenging to diagnose preoperatively due to its tendency to mimic conventional CCA. Unlike NET, where a favorable prognosis can be expected following surgical resection, NEC and MANEC were associated with poor clinical outcomes. Furthermore, old age ( $>60$ years) and the presence of tumor recurrence were associated with a decreased survival rate.

\section{Acknowledgements}

Not applicable.

\section{Funding}

This study was supported by grants from the National Natural Science Foundation of China (grant no. 31671019) and the National S\&T Major Project (grant no. 2017ZX10203205). 


\section{Availability of data and materials}

The datasets used and/or analyzed during the current study are available from the corresponding author on reasonable request.

\section{Authors' contributions}

SZ assessed the clinical findings of the case and was responsible for the conception of the study. HX, LZ and ML designed the research and were responsible for quality control of data. ZY, QC, DW and XZ made substantial contributions to acquisition, analysis and interpretation of data. LZ, ZY and QC wrote the manuscript. HX and SZ provided constructive discussions and revised the manuscript critically for important intellectual content. All authors have read and approved the final manuscript.

\section{Ethics approval and consent to participate}

Not applicable.

\section{Patient consent for publication}

Written informed consent for publication of this case report was obtained from the patient.

\section{Competing interests}

The authors declare that they have no competing interests.

\section{References}

1. Modlin IM, Oberg K, Chung DC, Jensen RT, de Herder WW, Thakker RV, Caplin M, Delle Fave G, Kaltsas GA, Krenning EP, et al: Gastroenteropancreatic neuroendocrine tumours. Lancet Oncol 9: 61-72, 2008.

2. Uccella S, La Rosa S, Volante $M$ and Papotti $M$ : Immunohistochemical biomarkers of gastrointestinal, pancreatic, pulmonary, and thymic neuroendocrine neoplasms. Endocr Pathol 29: 150-168, 2018.

3. Bosman FT, Carneiro F, Hruban RH and Theise ND: WHO Classification of Tumours of the Digestive System. Fourth Edition. IARC, Lyon, 2010.

4. La Rosa S, Marando A, Sessa F and Capella C: Mixed adenoneuroendocrine carcinomas (MANECs) of the gastrointestinal tract: An update. Cancers (Basel) 4: 11-30, 2012.

5. Vakili K and Pomfret EA: Biliary anatomy and embryology. Surg Clin North Am 88: 1159-1174, vii, 2008.

6. Rizvi S, Khan SA, Hallemeier CL, Kelley RK and Gores GJ: Cholangiocarcinoma-evolving concepts and therapeutic strategies. Nat Rev Clin Oncol 15: 95-111, 2018.

7. Yao JC, Hassan M, Phan A, Dagohoy C, Leary C, Mares JE, Abdalla EK, Fleming JB, Vauthey JN, Rashid A and Evans DB: One hundred years after 'carcinoid': Epidemiology of and prognostic factors for neuroendocrine tumors in 35,825 cases in the United States. J Clin Oncol 26: 3063-3072, 2008.

8. Michalopoulos N, Papavramidis TS, Karayannopoulou G, Pliakos I, Papavramidis ST and Kanellos I: Neuroendocrine tumors of extrahepatic biliary tract. Pathol Oncol Res 20: 765-775, 2014.

9. Sasatomi E, Nalesnik MA and Marsh JW: Neuroendocrine carcinoma of the extrahepatic bile duct: Case report and literature review. World J Gastroenterol 19: 4616-4623, 2013.

10. Zhang L, Wan D, Bao L, Chen Q, Xie H, Xu S and Lin S: Neuroendocrine carcinoma in the extrahepatic biliary tract: A case report and literature review. Medicine (Baltimore) 97: e11487, 2018

11. Acosta AM and Wiley EL: Primary biliary mixed adenoneuroendocrine carcinoma (MANEC): A short review. Arch Pathol Lab Med 140: 1157-1162, 2016.
12. Adsay V: Ki67 labeling index in neuroendocrine tumors of the gastrointestinal and pancreatobiliary tract: To count or not to count is not the question, but rather how to count. Am J Surg Pathol 36: 1743-1746, 2012.

13. Izumo W, Higuchi R, Yazawa T, Uemura S, Matsunaga Y, Shiihara M, Furukawa T and Yamamoto M: A long-term recurrence-free survival of a patient with the mixed adenoneuroendocrine bile duct carcinoma: A case report and review of the literature. Int J Surg Case Rep 39: 43-50, 2017.

14. Komo T, Kohashi T, Nakashima A, Ohmori I, Hihara J, Mukaida H, Kaneko M and Hirabayashi N: Mixed adenoneuroendocrine carcinoma of the distal bile duct: A case report. Int J Surg Case Rep 39: 203-207, 2017.

15. Lee SW, Lee IS, Cho YK, Park JM, Kim SW, Choi MG, Choi KY, Lee MA, Hong TH, You YK and Jung ES: A case of mixed adenoneuroendocrine carcinoma of the common bile duct: Initially diagnosed as cholangiocarcinoma. Korean J Pathol 48: 445-448, 2014.

16. Linder R, Dorfman T, Ben-Ishay O, Kakiashvili E, Velodavsky E and Kluger Y: Mixed neuroendocrine tumor of the common bile duct. JOP 14: 71-73, 2013.

17. Masui T, Doi R, Kawaguchi Y, Iwanaga Y, Ito T, Koizumi M and Uemoto S: Adenoendocrine cell carcinoma of the extrahepatic bile duct: A case report and review of the literature. Clin J Gastroenterol 4: 174-178, 2011.

18. Onishi I, Kitagawa H, Harada K, Maruzen S, Sakai S, Makino I, Hayashi $\mathrm{H}$, Nakagawara $\mathrm{H}$, Tajima $\mathrm{H}$, Takamura $\mathrm{H}$, et al: Intraductal papillary neoplasm of the bile duct accompanying biliary mixed adenoneuroendocrine carcinoma. World J Gastroenterol 19: 3161-3164, 2013.

19. Priyanka Akhilesh S, Kamal Sunder Y, Chandralekha T, Samir P and Prasad Kashinath W: Common hepatic duct mixed adenoneuroendocrine carcinoma masquerading as cholangiocarcinoma. Case Rep Gastrointest Med 2016: 4827050, 2016.

20. Wysocki J, Agarwal R, Bratton L, Nguyen J, Weidenhaft MC, Shores N and Kimbrell HZ: Mixed large cell neuroendocrine carcinoma and adenocarcinoma with spindle cell and clear cell features in the extrahepatic bile duct. Case Rep Pathol 2014: 347949, 2014.

21. Dancygier H, Klein U, Leuschner U, Hübner K and Classen M: Somatostatin-containing cells in the extrahepatic biliary tract of humans. Gastroenterology 86: 892-896, 1984.

22. Oshiro H, Matsuo K, Mawatari H, Inayama Y, Yamanaka S, Nagahama K, Endo I, Shimada H, Nakajima A and Kubota K: Mucin-producing gallbladder adenocarcinoma with focal small cell and large cell neuroendocrine differentiation associated with pancreaticobiliary maljunction. Pathol Int 58: 780-786, 2008.

23. Harada K, Sato Y, Ikeda H, Maylee H, Igarashi S, Okamura A, Masuda S and Nakanuma Y: Clinicopathologic study of mixed adenoneuroendocrine carcinomas of hepatobiliary organs. Virchows Arch 460: 281-289, 2012.

24. Harada K, Sato Y, Ikeda H, Hsu M, Igarashi S and Nakanuma Y: Notch1-Hes1 signalling axis in the tumourigenesis of biliary neuroendocrine tumours. J Clin Pathol 66: 386-391, 2013.

25. Mia-Jan K, Munkhdelger J, Lee MR, Ji SY, Kang TY, Choi E and Cho MY: Expression of CD133 in neuroendocrine neoplasms of the digestive tract: A detailed immunohistochemical analysis. Tohoku J Exp Med 229: 301-309, 2013.

26. Scardoni M, Vittoria E, Volante M, Rusev B, Bersani S, Mafficini A, Gottardi M, Giandomenico V, Malleo G, Butturini G, et al: Mixed adenoneuroendocrine carcinomas of the gastrointestinal tract: Targeted next-generation sequencing suggests a monoclonal origin of the two components. Neuroendocrinology 100: 310-316, 2014.

27. Lewin K: Carcinoid tumors and the mixed (composite) glandular-endocrine cell carcinomas. Am J Surg Pathol 11 (Suppl 1): S71-S86, 1987.

28. Liu Z, Zhang DY, Lu Z, Zhang P, Sun WL, Ma X, Wu H, Wu BQ and Zhou S: Neuroendocrine tumor of the common bile duct: A case report and review of the literature. Onco Targets Ther 11: 2295-2301, 2018

29. Squillaci S, Marchione R, Piccolomini M, Colombo F, Bucci F, Bruno $\mathrm{M}$ and Bisceglia $\mathrm{M}$ : Well-differentiated neuroendocrine carcinoma (malignant carcinoid) of the extrahepatic biliary tract: Report of two cases and literature review. APMIS 118: 543-556, 2010.

30. Zhan J, Bao G, Hu X, Gao W, Ruo X, Gong J, Zhu Q and Liu Y: Carcinoid tumor of the common bile duct in children: A case report. J Pediatr Surg 45: 2061-2063, 2010.

31. Cappell MS, Killeen TC and Jury R: Common bile duct carcinoid mimicking the clinical, EUS, and ERCP findings of cholangiocarcinoma: A rare but potentially curable cause of obstructive jaundice. Clin Gastroenterol Hepatol 9: e112-e113, 2011. 
32. Lee JH, Lee KG, Oh YH, Paik SS, Park HK and Lee KS: Carcinoid tumors of the extrahepatic biliary tract: Report of four cases. Surg Today 41: 430-435, 2011.

33. Bhalla P, Powle V, Shah RC and Jagannath P: Neuroendocrine tumor of common hepatic duct. Indian J Gastroenterol 31: 144-146, 2012.

34. De Luca L, Tommasoni S, de Leone A, Bianchi ML, de Nictolis M and Baroncini D: Neuroendocrine tumor of the extrahepatic bile duct: A tumor in an unusual site visualized by cholangioscopy. Endoscopy 45 (Suppl 2): E338-E339, 2013.

35. Jethava A, Muralidharan V, Mesologites T, Stoica-Mustafa E and Dasanu CA: An unusual presentation of a carcinoid tumor of the common bile duct. JOP 14: 85-87, 2013.

36. Yasuda T, Imai G, Takemoto M, Yamasaki M, Ishikawa $H$, Kitano M, Nakai T and Takeyama Y: Carcinoid tumor of the extrahepatic bile duct: Report of a case. Clin J Gastroenterol 6: 177-187, 2013.

37. Ayllon-Teran MD, Valverde-Martinez A, Diaz-Nieto R, Ciria-Bru R, Luque-Molina A, López-Cillero $\mathrm{P}$ and Briceño-Delgado J: Carcinoid tumor of the common bile duct. Rev Esp Enferm Dig 106: 560-561, 2014.

38. Khuroo S, Rashid A, Bali RS, Mushtaque M and Khuroo F: Carcinoid Klatskin tumour: A rare cause of obstructive jaundice. Australas Med J 7: 243-246, 2014.

39. Park SB, Moon SB, Ryu YJ, Hong J, Kim YH, Chae GB and Hong SK: Primary large cell neuroendocrine carcinoma in the common bile duct: First Asian case report. World J Gastroenterol 20: 18048-18052, 2014.

40. Yalav O, Ulku A, Demiryurek H and Doran F: A rare cause of bile duct obstruction in adolescence: Neuroendocrine tumor. Turk J Gastroenterol 25 (Suppl 1): S311-S312, 2014.

41. Kihara Y, Yokomizo H, Urata T, Nagamine M and Hirata T: A case report of primary neuroendocrine carcinoma of the perihilar bile duct. BMC Surg 15: 125, 2015.

42. Banerjee JK, Saranga Bharathi R, Shrivastava S and Ranjan P: Neuroendocrine tumor of distal bile duct. Med J Armed Forces India 72 (Suppl 1): S101-S104, 2016.

43. Hosoda K, Kobayashi A, Shimizu A, Kitagawa N, Ito T, Yamada A and Miyagawa S: Neuroendocrine tumor of the common bile duct. Surgery 160: 525-526, 2016.

44. Murakami M, Katayama K, Kato S, Fujimoto D, Morikawa M, Koneri K, Hirono Y and Goi T: Large-cell neuroendocrine carcinoma of the common bile duct: A case report and a review of literature. Surg Case Rep 2: 141, 2016

45. Oshiro Y, Gen R, Hashimoto S, Oda T, Sato T and Ohkohchi N: Neuroendocrine carcinoma of the extrahepatic bile duct: A case report. World J Gastroenterol 22: 6960-6964, 2016.

46. Raspanti C, Falco N, Silvestri V, Rotolo G, Bonventre S and Gulotta G: Neuroendocrine tumor of the common bile duct: Case report. G Chir 37: 275-280, 2016.

47. Abe T, Nirei A, Suzuki N, Todate Y, Azami A, Waragai M, Sato A, Takano Y, Nishino N, Sakuma H and Teranishi Y: Neuroendocrine tumor of the extrahepatic bile duct: A case report. Int J Surg Case Rep 40: 6-9, 2017.

48. Costin AI, Păun I, Păun M, Constantin VD and Vârcuş F: Primary neuroendocrine tumors-an extremely rare cause of obstruction of extrahepatic bile ducts: A case report. Rom J Morphol Embryol 58: 641-644, 2017.

49. Hoepfner L and White JA: Primary extrahepatic bile duct neuroendocrine tumor with obstructive jaundice masquerading as a Klatskin tumor. J Surg Case Rep 2017: rjx 104, 2017.

50. Khan FA, Stevens-Chase A, Chaudhry R, Hashmi A, Edelman D and Weaver D: Extrahepatic biliary obstrution secondary to neuroendocrine tumor of the common hepatic duct. Int J Surg Case Rep 30: 46-49, 2017.
51. Sanchez Cabus S, Pittau G, Sebagh M and Cherqui D: Primary non-functioning neuroendocrine tumor of the extrahepatic bile duct. Rev Esp Enferm Dig 109: 228-229, 2017.

52. Sano I, Kuwatani M, Sugiura R, Kato S, Kawakubo K, Ueno T, Nakanishi Y, Mitsuhashi T, Hirata H, Haba S, et al: Hepatobiliary and pancreatic: A rare case of a well-differentiated neuroendocrine tumor in the bile duct with spontaneous regression diagnosed by EUS-FNA. J Gastroenterol Hepatol 32: 11, 2017.

53. Koo JY, Kim KH and Kim TN: Primary large cell neuroendocrine carcinoma of the common hepatic duct mimicking a Klatskin tumor. Korean J Intern Med 34: 452-453, 2019.

54. Oberg K, Knigge U, Kwekkeboom D and Perren A; ESMO Guidelines Working Group: Neuroendocrine gastro-entero-pancreatic tumors: ESMO clinical practice guidelines for diagnosis, treatment and follow-up. Ann Oncol 23 (Suppl 7): vii124-vii130, 2012.

55. Hong N, Kim HJ, Byun JH, Kim SY, Kim KW, Kim JH and Hong SM: Neuroendocrine neoplasms of the extrahepatic bile duct: Radiologic and clinical characteristics. Abdom Imaging 40: 181-191, 2015.

56. Sundin A, Arnold R, Baudin E, Cwikla JB, Eriksson B, Fanti S, Fazio N, Giammarile F, Hicks RJ, Kjaer A, et al: ENETS consensus guidelines for the standards of care in neuroendocrine tumors: Radiological, nuclear medicine \& hybrid imaging. Neuroendocrinology 105: 212-244, 2017.

57. Perren A, Couvelard A, Scoazec JY, Costa F, Borbath I, Delle Fave G, Gorbounova V, Gross D, Grossma A, Jense RT, et al: ENETS consensus guidelines for the standards of care in neuroendocrine tumors: Pathology: Diagnosis and prognostic stratification. Neuroendocrinology 105: 196-200, 2017.

58. Kaltsas G, Caplin M, Davies P, Ferone D, Garcia-Carbonero R, Grozinsky-Glasberg S, Hörsch D, Tiensuu Janson E, Kianmanesh R, Kos-Kudla B, et al: ENETS consensus guidelines for the standards of care in neuroendocrine tumors: Pre- and perioperative therapy in patients with neuroendocrine tumors. Neuroendocrinology 105: 245-254, 2017.

59. Sorbye H, Welin S, Langer SW, Vestermark LW, Holt N, Osterlund P, Dueland S, Hofsli E, Guren MG, Ohrling K, et al: Predictive and prognostic factors for treatment and survival in 305 patients with advanced gastrointestinal neuroendocrine carcinoma (WHO G3): The NORDIC NEC study. Ann Oncol 24: 152-160, 2013

60. Brathwaite S, Yearsley MM, Bekaii-Saab T, Wei L, Schmidt CR, Dillhoff ME, Frankel WL, Hays JL, Wu C and Abdel-Misih S: Appendiceal mixed Adeno-Neuroendocrine carcinoma: A Population-based study of the surveillance, epidemiology, and end results registry. Front Oncol 6: 148, 2016.

61. Rayhan N, Sano T, Qian ZR, Obari AK and Hirokawa M: Histological and immunohistochemical study of composite neuroendocrine-exocrine carcinomas of the stomach. J Med Invest 52: 191-202, 2005.

62. La Rosa S, Marando A, Furlan D, Sahnane N and Capella C: Colorectal poorly differentiated neuroendocrine carcinomas and mixed adenoneuroendocrine carcinomas: Insights into the diagnostic immunophenotype, assessment of methylation profile, and search for prognostic markers. Am J Surg Pathol 36: 601-611, 2012.

This work is licensed under a Creative Commons Attribution-NonCommercial-NoDerivatives 4.0 International (CC BY-NC-ND 4.0) License. 